\title{
The resurgence of neurotransmitter modulation in Parkinson's disease with safinamide
}

This article was published in the following Dove Press journal:

Journal of Parkinsonism and Restless Legs Syndrome

26 March 2015

Number of times this article has been viewed

\section{Thomas Müller}

Department of Neurology, St Joseph Hospital Berlin-Weißensee, Berlin, Germany
Correspondence: Thomas Müller Department of Neurology, St Joseph Hospital Berlin-Weißensee, Gartenstr I, I 3088 Berlin, Germany

Tel +493092790223

Fax +493092790703

Email th.mueller@alexius.de; thomas. mueller@ruhr-uni-bochum.de

\begin{abstract}
The main feature of Parkinson's disease is slowly ongoing neuronal death. Changes of neurotransmission of biogenic amines, such as dopamine, cause the heterogeneity of motor and non-motor symptoms. Therefore, compounds with a broad spectrum of mechanisms of action are ideal candidates for the treatment of the disease. Safinamide reduces dopamine turnover by reversible monoamine oxidase B inhibition, blockage of voltage-dependent sodium channels, and modulation of calcium channels and of glutamate release. Safinamide requires one-time daily intake within a dose range of 50 and $100 \mathrm{mg}$. Clinical trials demonstrated that safinamide is well tolerated and safe and ameliorates motor behavior when combined with dopamine agonist only or dopamine agonist and levodopa. Safinamide is a putative, important drug for the therapy of Parkinson's disease with an efficacy superior to available irreversible monoamine oxidase B inhibitors or N-methyl-D-aspartate receptor antagonists.
\end{abstract}

Keywords: MAO-B inhibition, glutamate release inhibition, dopamine substitution, glutamate

\section{Introduction}

Parkinson's disease (PD) is the second most common chronic neurodegenerative disorder. It also affects movement behavior. Two percent of individuals over age 65 years and up to $5 \%$ over 85 years suffer from this disease. ${ }^{1} \mathrm{PD}$ is mainly pathologically characterized by dopaminergic neuronal loss in the substantia nigra and consecutively by striatal dopamine loss with the accumulation of the protein $\alpha$-synuclein. ${ }^{2-4}$ However, chronic neuronal death also affects other neurotransmitter systems both in the periphery and the brain. In the periphery, orthostatic hypotension is a result of sympathetic neurocirculatory failure. In the brain, the neurodegenerative process also takes place in the predominant norepinephrinergic locus ceruleus, the serotonergic raphe nuclei, and the cholinergic nucleus basalis of Meynert. This kind of chronic neurodegeneration induces dysfunction of cortical and limbic projections and disturbances of vegetative nervous system function in the region of the dorsal nucleus of the vagus nerve or the sympathetic ganglia. ${ }^{2-4}$ Additionally, serious cytoskeletal damage is found in glutamatergic, gamma aminobutyric acidergic, cholinergic, noradrenergic, serotonergic, and peptidergic neurons. ${ }^{5}$ As a result, an individually pronounced and different expression of symptoms occurs in each PD patient. Tremor at rest, bradykinesia, rigidity, and postural instability are the main features of motor impairment. These symptoms are accomplished by an onset of a wide variety of non-motor features as further characteristics of the disease. ${ }^{6-8}$ The term PD actually reflects a superordinate concept for a variety of different kinds of diseases. They resemble each other and do not always share the concept of the neu- 
ropathological manifestation of Lewy bodies as an essential initial step to the onset of the disease process. ${ }^{4,9,10}$

\section{The unknown cause of PD}

Various hypotheses about the cause of PD discuss genetic defects or gene mutations; impaired detoxification capacity; exposure to acute and chronic endogenous and exogenous toxins, such as pesticides; deficiencies of mitochondrial function; infection by prion-like proteins; protein misfolding; inflammation; and decrease of neurotransmitter capacity, including monoamine storage vesicles and glutamate metabolism. ${ }^{11-13}$ All the events within the neuronal cell death cascade are characterized by one common step, which is an increased synthesis of free radicals, contributing to neuronal death in the end. All these complementing mechanisms contribute to onset of the heterogeneous forms of PD as a result of the predominant death of dopamine-synthesizing presynaptic nigrostriatal neurons. ${ }^{5,11-13}$

\section{Drawbacks for cure or very specific-acting drugs in PD}

This heterogeneity of clinical symptoms in PD requires an individually adapted treatment regime. Development of drug targets based on molecular and genetic research and attempts to cure PD or to delay progression of PD by diseasemodifying, neuroprotective, neuroregenerative therapies have more or less failed in past years. ${ }^{14,15}$ These disappointments in PD drug research are caused by various obvious but only partially scrutinized reasons. They may complement each other. The etiology of sporadic forms of PD is still unknown and various mechanisms of neuronal death occur. Accordingly, various types of neuronal cells degenerate in an individually different and pronounced fashion in various areas of the brain. Findings from genetic association studies or gene mutations did not reflect the variety of the different subtypes of PD observed in the clinic. Accordingly developed curing approaches more or less failed in experimental studies or later in the clinic. Environmental surroundings, chronic intoxication, or chronic infection with still-unknown causative organisms may play an eminent role in genetically predisposed individuals for the final onset of PD. Moreover, the in vitro and in vivo models of PD are overestimated in experimental research. Most of these models do not even reflect the chronic nature of slow ongoing neuronal cell death in various neurons, since, for example, toxin models, ie, 6-OH-dopamine, rotenone, or 1-methyl-4-phenyl-1,2,3,6tetrahydropyridine application, generate an acute event associated with a one-time toxin application. These models also only focus on the death of dopamine-synthesizing neurons in the nigrostriatal system. However, it is well known that the chronic neurodegenerative process in PD at least affects the whole brain and thus is also responsible for the onset of symptoms beyond the effects on motor behavior.

\section{Therapy}

There is a certain portfolio of drugs against motor and non-motor symptoms of PD. All of these are applied and titrated in an individual manner according to tolerability, safety, and the needs of the patients and their caregivers. Therefore, the bureaucratic development of standardized treatment approaches contradicts the inhomogeneous forms of PD in clinical practice. Guidelines for the treatment of PD should generally only be regarded as a very rough approach. They should only provide some general recommendations and should not go into too much detail. ${ }^{16}$ They should not only consider the fulfillment of evidence based medicine criteria. They often privilege placebo-controlled, randomized clinical studies performed according to the standard of good clinical practice. ${ }^{16}$ Most such trials have selected only a well-defined study population, which does not reflect the normal maintenance of PD patients. Real advances in the treatment of PD patients have been provided by observation and the courage of physicians and their patients to test a new treatment paradigm. This was the case when levodopa was initially applied by Birkmayer et $\mathrm{a}^{17}$ or when the motor symptom-enhancing effects of amantadine were observed during treatment of influenza. Nowadays, there is also a growing misuse of guideline recommendations. Health politicians and payers (ie, health insurers), employ them to accomplish budget restrictions for physicians in clinical practice with concomitant limitations of the therapy quality. This development is observed in more and more countries all over the world. Authors of guidelines should scrutinize them as to whether they really support the quality of treatment of PD patients and whether they are abused to enforce a reduction of health care costs, which mostly results from an administrative overload and not from pharmacotherapy. The real unmet medical need nowadays is that PD patients do not receive an appropriate therapy anymore; that more and more generic drugs with varying pharmacodynamic properties are given in too-low dosages. As an alternative, patients are switched to deep brain stimulation (DBS). DBS may reduce costs for pharmacotherapy to a certain extent in the medium term. Administrative health economic considerations may therefore support this DBS approach according to the following slogan: a high investment in DBS will reduce drug costs in 
the long-term. However, DBS provides a continuous balance with biogenic amines from the neurochemical point of view and might elevate the risk for personality changes and cognitive disturbances. ${ }^{18-20}$ Both clinical symptoms limit the use of expensive combination drug regimes. Instead, concomitant application of the cheap, traditional levodopa formulations is mainly tolerated by these DBS-treated PD patients in the long-term. However, one long-term consequence of DBS is often increased caregiver and nursing burden, which has not been reported so far within scientific studies, but from patient organizations. Thus, a real innovation in the drug therapy of PD has not occurred in past years. Many tested compounds mostly failed due to a diabolic combination of inappropriate designs, unrealistic demands of the approving authorities, application of wrong assessment tools, and probably too-high standards for the safety and tolerability. The most effective drug for the treatment of PD, levodopa, would not survive in the contemporary study and pharmaceutical world due to too-frequent onset of electrocardiogram changes and likely nausea and gastrointestinal disturbances. There are three main pharmacological principles of balancing the dopaminergic deficit in PD. The first one is application of the precursor of dopamine, levodopa, which only traverses the blood-brain barrier in contrast to dopamine itself. Levodopa has a short half-life, which causes fluctuations of levodopa plasma levels. These ups and downs are converted after the conversion of levodopa to dopamine in presynaptic tangles into a pulsatile stimulation of dopaminergic receptors, which clinically results in the onset of non-motor and motor complications, sometimes within months. The second principle is the direct stimulation of postsynaptic neurons by dopamine agonists. Their efficacy depends on the affinity to the dopaminergic uptake sites and their half-life. Thus, they delay onset of motor complications, but their acceptability suffers as a result of their side effect profile. This is predominantly characterized by nausea, edema, sleepiness, and various kinds of dopamine dysregulation syndrome, which probably results from a too-continuous stimulation of dopamine-sensitive mesolimbic structures. The third pharmacologic principle is the irreversible inhibition of the enzyme monoamine oxidase $\mathrm{B}$ (MAO-B), which degrades dopamine in glial cells and thus contributes to the free radical generation. As a result, a stabilization of dopamine concentrations in the synaptic cleft takes place. This prolongation of the dopamine efficacy provides a limited effect on motor symptoms. There are two compounds currently available, the generic selegiline and in Europe the soon-to-be generic, rasagiline. Rasagiline also has N-methylD-aspartate (NMDA) antagonistic properties, which may contribute to an indirect improvement of dopaminergic neurotransmission similar to the mode of action provided by amantadine. ${ }^{21}$ A similar compound, safinamide, is on the horizon, which only inhibits MAO-B in a reversible fashion and possesses NMDA antagonistic properties combined with a blockade of voltage-gated $\mathrm{Na}^{+}$channels. All these MAOB-inhibiting compounds have a better safety and tolerability profile than dopamine agonists and levodopa. ${ }^{21}$

\section{Safinamide}

Safinamide [(S)-2-((4-((3-fluorobenzyl)oxy)benzyl)amino) propanamide] is a small, water-soluble, chemical and metabolic stable compound. This drug has a low central nervous system toxicity. The essential mechanism of action of safinamide is the specific and potent modulation of dopamine metabolism. This effect is enabled by reversible MAO-B inhibition, which is different to the irreversible MAO-B inhibition and MAO-A inhibition observed in the clinic during the chronic application of selegiline and rasagiline. Safinamide was found to be approximately 5,000 times more selective for MAO-B than MAO-A in rats and 1,000 times more selective for MOA-B in humans. ${ }^{22,23}$ Selegiline is 127 times more selective for MAO-B than for MAO-A; rasagiline is 103 times more selective for MAO-B than for MAO-A in humans. ${ }^{24}$ Additionally, safinamide is a blocker of $\mathrm{Na}^{+} / \mathrm{Ca}^{2+}$ channels, which finally contributes to glutamate release inhibition. Safinamide is well absorbed, metabolized in a linear fashion, and has a long half-life. There are hints of possible disease-modifying neuroprotective features of safinamide, independent of MAO-B inhibition, according to experimental findings in in vitro and in vivo models for $\mathrm{PD}^{24}$ and for multiple sclerosis. ${ }^{25}$ Safinamide blocked in vitro veratridine-induced neuron cell death by $\mathrm{Na}^{+}$and $\mathrm{Ca}^{2+}$ channel inhibition, protected against hippocampal neuron loss from the glutamate analog kainic acid in rats, and protected against ischemia-induced hippocampal neuron death in gerbils. ${ }^{22-31}$ Safinamide applied in a dose range between 3 and $30 \mathrm{mg} / \mathrm{kg}$ reduced intensity, duration, and, accordingly, scores of levodopa-induced dyskinesia as well as prolonged the effect of levodopa on motor behavior. This effect was similar to that of amantadine applied in 5 and $20 \mathrm{mg} / \mathrm{kg}$ doses. Combined with amantadine $(5 \mathrm{mg} / \mathrm{kg})$, safinamide (3 $\mathrm{mg} / \mathrm{kg}$ ) did not enhance levodopa-induced dyskinesia, whereas $20 \mathrm{mg} / \mathrm{kg}$ of safinamide showed additional beneficial effects on dyskinesia and prevented a worsening of the duration of the levodopa effect on motor behavior, which was observed with amantadine only. The tremulous jaw movement model, an animal model of parkinsonian tremor, 
was employed to investigate putative tremor-reducing effects of safinamide. Tremulous jaw movements were induced following intraperitoneal application of the acetylcholinesterase inhibitor galantamine $(3.0 \mathrm{mg} / \mathrm{kg})$, of the muscarinic agonist pilocarpine $(0.5 \mathrm{mg} / \mathrm{kg})$, and of the dopamine $\mathrm{D}_{2}$ receptor antagonist pimozide ( $1 \mathrm{mg} / \mathrm{kg}$ ). Dosages of 5-10 mg/kg safinamide improved tremulous jaw movements induced by all three compounds. ${ }^{32,33}$ These experimental findings point out that safinamide shares some effects of the well-investigated antiparkinsonian efficacy of amantadine and budipine. The combination of reversible MAO-B inhibition and glutamate release inhibition supports the efficacy of this compound on motor symptom alleviation in PD patients and indicates a certain potential as an antidyskinetic drug in PD. However, it is hard to demonstrate antidyskinetic effects in randomized clinical trials in the long-term. ${ }^{34}$

\section{Safinamide in clinical trials}

Several trials, performed in PD patients, have demonstrated the efficacy, tolerability, and safety of safinamide. ${ }^{21}$

\section{Dose-finding study in early PD patients}

The 009 trial included 172 PD patients, two withdrew consent and two did not meet the inclusion criteria. Fifty-six patients were put into each group (treated or not treated with single dopamine agonist); of these, 49 completed the placebo arm, 52 the lower $(0.5 \mathrm{mg} / \mathrm{kg}$, equivalent to approximately $40 \mathrm{mg} /$ day), and 49 the higher $(1 \mathrm{mg} / \mathrm{kg}$ equivalent to approximately $90 \mathrm{mg}$ /day) safinamide dose arms. There were no significant differences between groups at baseline or concerning the withdrawal rate during the trial. One hundred and one patients were on dopamine agonists (apomorphine, one; bromocriptine, nine; cabergoline, eight; pergolide, 31; piribedil, four; pramipexole, 32; ropinirole, 16). The response to safinamide was defined as a $\geq 30 \%$ improvement in the Unified Parkinson's Disease Rating Scale part III (UPDRS III) scores at study end versus baseline. At the end, therapy with safinamide $(0.5 \mathrm{mg} / \mathrm{kg}$ dose: 17 responders, UPDRS III score difference to baseline: $2.6 ; 1 \mathrm{mg} / \mathrm{kg}$ dose: 21 responders, UPDRS III score difference to baseline: 3.3 ) was better than placebo (12 responders, UPDRS III score difference to baseline: 0.6 ) in the intention-to-treat cohort. A sub-analysis in dopamine agonist only-treated patients demonstrated that safinamide $(0.5 \mathrm{mg} / \mathrm{kg}$ dose: 12 responders, UPDRS III score difference to baseline: $4 ; 1 \mathrm{mg} / \mathrm{kg}$ dose: 16 responders, UPDRS III score difference to baseline: 4.7) was superior to placebo application (seven responders, UPDRS III score difference to baseline: 1.4$).{ }^{35}$

\section{Safinamide in early PD patients}

The 015 study was a 6-month, randomized, double-blind, placebo-controlled, international trial. ${ }^{36}$ This trial investigated the addition of safinamide once daily to a stable dose of a single dopamine agonist in 270 early PD patients, suffering less than 5 years from PD. Patients were not enrolled if they showed motor complications. Further causes for screening failures were the intake of more than one dopamine agonist or of any further PD drug in the 4 weeks preceding screening. Patients with dementia or cognitive dysfunction were not enrolled if the Mini-Mental State Examination (MMSE) score was $<24$ or if patients had a score of 3 on item 1 of UPDRS I. Finally, patients with serious medical conditions or with mental or physical conditions that would preclude collection of safety or efficacy data were also excluded. Participants were randomized to one of the three arms of the study to receive either safinamide at a dose of 50 to $100 \mathrm{mg}$ once daily (90 patients), safinamide at a dose of 150 to $200 \mathrm{mg}$ once daily (90 patients), or matching placebo tablets (90 patients), as an add-on treatment to dopamine agonist therapy. Coadministration of safinamide 50-100 mg/day improved motor symptoms. The UPDRS III score was superior to the effect of dopamine agonist monotherapy (difference between end of study and baseline of $-6.0 \pm 7.2$ in the safinamide-treated group versus $-3.6 \pm 7.1$ in the placebo group; $P=0.0419$. Accordingly, therapy with safinamide $50-100 \mathrm{mg}$ per day better ameliorated the UPDRS II score in comparison with dopamine agonist monotherapy (difference between end of study and baseline of $-2.2 \pm 3.8$ in the safinamide-treated group versus $-1.2 \pm 3.5$ in the placebo group; $P=0.0248$. The higher safinamide dose range of 150-200 mg/day was not superior to safinamide $50-100 \mathrm{mg}$ per day according to the rating with the total UPDRS. The higher safinamide dosing was related to an elevated rate of premature discontinuations in comparison with the low-dose and placebo groups. Thus, the 50-100 mg/day safinamide dose range was looked upon as the most appropriate dosing for further studies.

The patients of 01 could enter the 52-week extension phase trial 017 . Its primary endpoint was the interval to intervention, which was defined as the appearance of one of the following events: a necessary increase of the dopamine agonist dose; supplementation with another dopamine agonist, levodopa, or another antiparkinsonian drug; or cessation of treatment due to lack of efficacy. Data from both dose groups were pooled. They did not reach statistical significance for this primary endpoint, probably due to a failure of response observed in patients receiving the higher safinamide dose (150-200 mg/day). The outcomes of a post hoc analysis 
revealed that participants on 50-100 mg safinamide per day needed a lower rate of interventions compared with patients receiving dopamine agonist monotherapy (plus placebo) ( $25 \%$ versus $51 \% ; P=0.0479$ ). The secondary efficacy endpoint during the extension phase was defined as the mean change in UPDRS III scores in the pooled safinamide group. An analysis performed on UPDRS III score changes by treatment groups demonstrated that safinamide $50-100 \mathrm{mg}$ / day resulted in an amelioration of motor symptoms during the 18-month treatment period. The better motor behavior supported a better quality of life according to the outcomes of the EuroQol-5D (EQ-5D) scale. ${ }^{37}$

\section{The MOTION study}

This Phase III trial ${ }^{38}$ showed that combination of $50 \mathrm{mg}$ or $100 \mathrm{mg}$ safinamide with one dopamine agonist only is a welltolerated and safe treatment option in PD. Six hundred and seven out of 679 randomized patients finished the 24-week treatment interval. At the end, 13 patients were excluded, since the clinical research organization noted at that point that these participants did not meet the predefined inclusion criteria due to additional PD drug intake or missing dopamine agonist therapy during the inclusion process. Therefore, a modified analysis of the intention-to-treat patient group was done in order to compute the results in an adequate manner. Patients with intake of a single dopamine agonist (666 patients) showed a benefit of safinamide $100 \mathrm{mg}$ /day $(P=0.04)$ in comparison with placebo. Their motor symptoms improved according to the UPDRS III ( -2.06 [mean change]; 95\% CI: $[-2.35 ;-0.06])$ results. PD patients on additional $50 \mathrm{mg}$ safinamide per day only showed a reduction of UPDRS III scores (-1.93 [mean change]; 95\% CI: [-1.85; 0.44]), but this did not significantly differ from the results with placebo therapy. The EQ-5D outcomes confirmed this $(P=0.0207)$ improvement for safinamide $100 \mathrm{mg}$ /day in comparison with placebo (difference versus placebo: 0.039 [mean]; 95\% CI: $[0.011 ; 0.068])$. There was no significant difference for the safinamide $50 \mathrm{mg}$ /day arm compared with the placebo treatment. A significant improvement was also observed for safinamide $100 \mathrm{mg}$ per day versus placebo in the 39-item Parkinson's Disease Questionnaire (PDQ-39) index scores, whereas no significant differences were shown for the safinamide $50 \mathrm{mg}$ /day group in comparison with placebo. ${ }^{38}$

\section{Safinamide in advanced PD patients}

The 016 study was a global Phase III trial with a 6-month duration. Six hundred and sixty-nine advanced PD patients, suffering from PD for at least 3 years, took either safinamide or placebo. They were on a stable levodopa treatment and had "off" periods of 1.5 hours. A concomitant stable therapy with a dopamine agonist and/or an anticholinergic drug was necessary for inclusion. There was a 4-week-long period for a stable titration of the levodopa dosing. Then, participants were randomized to one of the three treatment arms on a 1:1:1 basis. They received one of two different doses of safinamide ( 50 or $100 \mathrm{mg}$ once daily: 223 and 224 patients, respectively) or placebo (222 patients) in combination with their remaining drug regimen including levodopa. The primary endpoint was the increase of daily "on" time (on time with and without minor dyskinesia) during an 18-hour period. The on time was evaluated by the daily diary records of the patients. Intakes of $50 \mathrm{mg}$ and $100 \mathrm{mg}$ respectively safinamide increased the daily total on time at the end of this trial, after 6 months. This difference turned out to be significant ( $P=0.022$ [safinamide $50 \mathrm{mg}$ ] respectively $P=0.013$ [safinamide $100 \mathrm{mg}$ ]). There was no increase of on time with troublesome dyskinesia. Accordingly, the UPDRS IV scores were lowered by both doses of safinamide. This reduction was significant in the patients with $100 \mathrm{mg}$ safinamide per day. The $100 \mathrm{mg}$ dose improved the emotional well-being as evaluated by a subscale of the PDQ-39. Out of the 669 randomized patients, $89 \%$ of patients treated with safinamide finished the trial $(91 \%$ in the $50 \mathrm{mg}$ dose group and $87 \%$ in the $100 \mathrm{mg}$ dose group) in comparison with the $89 \%$ on placebo. Secondary efficacy endpoints of this study were also positive. They included decrease in daily off time, reduction of mean off time following the first morning dose of levodopa, and improvement of the UPDRS III score during on time and the Clinical Global Impression of Severity score from baseline. Ninety percent of the 016 study patients entered a 78-week, placebo-controlled, double-blind extension study, which looked at the effect on dyskinesias as a primary endpoint. This extension study did not reach this primary endpoint. ${ }^{21,39,40}$

\section{The SETTLE study}

This trial investigated the efficacy of safinamide addition after prior titration to a stable dosing regimen of the PD drug therapy in PD patients with fluctuations of movement within 4 weeks. After stabilization, the 549 participants additionally took $50 \mathrm{mg}$ or $100 \mathrm{mg}$ safinamide or placebo for 24 weeks. Four hundred and eighty-four of them finished this Phase III trial. Patients on either 50 or $100 \mathrm{mg}$ safinamide experienced less off time (1.03 [mean]; 95\% CI: [-1.40; -0.67] hours), more on time (0.96 [mean]; 95\% CI: [0.56; 1.37] hours), and better UPDRS III scores during on time $(-1.82$ [mean]; 95\% CI: $[-3.01 ;-0.62])$ in comparison with the placebo application. ${ }^{40}$ 
Accordingly, the scores of PDQ-39 (-2.33 [mean]; 95\% CI: [-3.98; -0.68$])$ and the EQ-5D (0.06 [mean]; 95\% CI: [0.03; $0.09])$ were better than in the placebo group. ${ }^{21,41}$

\section{Safety and tolerability of safinamide}

Safinamide was administered to healthy male volunteers within a dose range of 25 to $10,000 \mu \mathrm{g} / \mathrm{mL}$. There was a linear pharmacokinetic behavior, proportionally associated with the administered dose. The clearance of safinamide from the body was half-life $=22$ hours, and no clinically relevant accumulation was found. Tolerability was good. In the clinical trials on PD patients, dosing of safinamide up to $200 \mathrm{mg} /$ day was well tolerated. No serious adverse events were described. Reported serious adverse events to date were not related to study medication.

\section{Conclusion}

This narrative review describes the available data on safinamide in the context of current therapies in PD. Safinamide was recently recommended by the Committee for Medicinal Products for Human Use as a precondition for recommendation by the European Medicines Agency. The European Medicines Agency gave their approval February 26, 2015. This compound is a selective and reversible MAO-B inhibitor, which increases levels of available dopamine and other biogenic amines in PD patients. Safinamide blocks sodium channels and modulates $\mathrm{Ca}^{2+}$ channels. This inhibits the release of glutamate. Safinamide has been evaluated as an adjunctive therapy to a dopamine agonist in early PD and to levodopa in mid-to-late PD patients with a positive outcome. ${ }^{21}$ Clinical trials have demonstrated the efficacy and tolerability of safinamide. The results of these studies suggest an optimum dose range of 50-100 mg/day. Therapy with safinamide may provide symptomatic benefits in PD and may also represent a step back to a more clinical therapeutic approach based on altered neurochemistry in the brain in PD.

\section{Disclosure}

The author participated in the trials on safinamide as principal investigator and gave advice to the following safinamidedeveloping pharmaceutical companies: Newron, MerckSerono, and Zambon. The author reports no other conflicts of interest in this work.

\section{References}

1. Rajput AH, Birdi S. Epidemiology of Parkinson's disease. Parkinsonism Relat Disord. 1997;3:175-186.

2. Brooks DJ. Examining Braak's hypothesis by imaging Parkinson's disease. Mov Disord. 2010;25 Suppl 1:S83-S88.

3. Hughes AJ, Daniel SE, Kilford L, Lees AJ. Accuracy of clinical diagnosis of idiopathic Parkinson's disease: a clinico-pathological study of 100 cases. J Neurol Neurosurg Psychiatry. 1992;55:181-184.
4. Milber JM, Noorigian JV, Morley JF, et al. Lewy pathology is not the first sign of degeneration in vulnerable neurons in Parkinson disease. Neurology. 2012;79:2307-2314.

5. Przuntek H, Müller T, Riederer P. Diagnostic staging of Parkinson's disease: conceptual aspects. J Neural Transm. 2004;111:201-216.

6. Lim SY, Fox SH, Lang AE. Overview of the extranigral aspects of Parkinson disease. Arch Neurol. 2009;66:167-172.

7. Lim SY, Lang AE. The nonmotor symptoms of Parkinson's disease - an overview. Mov Disord. 2010;25 Suppl 1:S123-S130.

8. Siderowf A, Lang AE. Premotor Parkinson's disease: concepts and definitions. Mov Disord. 2012;27:608-616.

9. Dickson DW. Parkinson's disease and parkinsonism: neuropathology. Cold Spring Harb Perspect Med. 2012;2(8):a009258.

10. Weiner WJ. There is no Parkinson disease. Arch Neurol. 2008;65: 705-708.

11. Blandini F. Neural and immune mechanisms in the pathogenesis of Parkinson's disease. J Neuroimmune Pharmacol. 2013;8:189-201.

12. Halliwell B. Role of free radicals in the neurodegenerative diseases: therapeutic implications for antioxidant treatment. Drugs Aging. 2001;18:685-716.

13. Naoi M, Maruyama W, Yi H, Inaba K, Akao Y, Shamoto-Nagai M. Mitochondria in neurodegenerative disorders: regulation of the redox state and death signaling leading to neuronal death and survival. J Neural Transm. 2009;116:1371-1381.

14. Lang AE, Gill S, Patel NK, et al. Randomized controlled trial of intraputamenal glial cell line-derived neurotrophic factor infusion in Parkinson disease. Ann Neurol. 2006;59:459-466.

15. Olanow CW, Goetz CG, Kordower JH, et al. A double-blind controlled trial of bilateral fetal nigral transplantation in Parkinson's disease. Ann Neurol. 2003;54:403-414.

16. Müller T. Drug therapy in patients with Parkinson's disease. Transl Neurodegener. 2012;1(1):10.

17. Birkmayer W, Hornykiewicz O. The effect of 1-3,4-dihydroxyphenylalanine (= DOPA) on akinesia in parkinsonism. 1961. Wien Klin Wochenschr. 2001;113(22);851-854.

18. Harati A, Müller T. Neuropsychological effects of deep brain stimulation for Parkinson's disease. Surg Neurol Int. 2013;4:S443-S447.

19. Figee $M$, de Koning $P$, Klaassen $S$, et al. Deep brain stimulation induces striatal dopamine release in obsessive-compulsive disorder. Biol Psychiatry. 2014;75:647-652.

20. Meissner W, Reum T, Paul G, et al. Striatal dopaminergic metabolism is increased by deep brain stimulation of the subthalamic nucleus in 6-hydroxydopamine lesioned rats. Neurosci Lett. 2001;303:165-168.

21. Müller T. Current status of safinamide for the drug portfolio of Parkinson's disease therapy. Expert Rev Neurother. 2013;13:969-977.

22. Caccia C, Maj R, Calabresi M, et al. Safinamide: from molecular targets to a new anti-Parkinson drug. Neurology. 2006;67:S18-S23.

23. Fariello RG. Safinamide. Neurotherapeutics. 2007;4:110-116.

24. Binda C, Wang J, Pisani L, et al. Structures of human monoamine oxidase B complexes with selective noncovalent inhibitors: safinamide and coumarin analogs. J Med Chem. 2007;50:5848-5852.

25. Morsali D, Bechtold D, Lee W, et al. Safinamide and flecainide protect axons and reduce microglial activation in models of multiple sclerosis. Brain. 2013;136:1067-1082.

26. Cattaneo C, Caccia C, Marzo A, Maj R, Fariello RG. Pressor response to intravenous tyramine in healthy subjects after safinamide, a novel neuroprotectant with selective, reversible monoamine oxidase B inhibition. Clin Neuropharmacol. 2003;26:213-217.

27. Chazot PL. Safinamide (Newron Pharmaceuticals). Curr Opin Investig Drugs. 2001;2:809-813.

28. Fariello RG, McArthur RA, Bonsignori A, et al. Preclinical evaluation of PNU-151774E as a novel anticonvulsant. J Pharmacol Exp Ther. 1998;285:397-403.

29. Stocchi F, Vacca L, Grassini P, et al. Symptom relief in Parkinson disease by safinamide: biochemical and clinical evidence of efficacy beyond MAO-B inhibition. Neurology. 2006;67:S24-S29. 
30. Strolin Benedetti MS, Marrari P, Colombo M, et al. The anticonvulsant FCE 26743 is a selective and short-acting MAO-B inhibitor devoid of inducing properties towards cytochrome P450-dependent testosterone hydroxylation in mice and rats. J Pharm Pharmacol. 1994;46: 814-819.

31. Strolin Benedetti M, Tocchetti P, Rocchetti M, et al. Enantioselective recognition of two anticonvulsants, FCE 26743 and FCE 28073, by MAO, and relationship between MAO-B inhibition and FCE 26743 concentrations in rat brain. Prog Brain Res. 1995;106:123-134.

32. Grégoire L, Jourdain VA, Townsend M, Roach A, Di Paolo T. Safinamide reduces dyskinesias and prolongs L-DOPA antiparkinsonian effect in parkinsonian monkeys. Parkinsonism Relat Disord. 2013;19: 508-514.

33. Podurgiel S, Collins-Praino LE, Yohn S, et al. Tremorolytic effects of safinamide in animal models of drug-induced parkinsonian tremor. Pharmacol Biochem Behav. 2013;105:105-111.

34. Goetz CG, Damier P, Hicking C, et al. Sarizotan as a treatment for dyskinesias in Parkinson's disease: a double-blind placebo-controlled trial. Mov Disord. 2007;22:179-186.

35. Stocchi F, Arnold G, Onofrj M, et al; Safinamide Parkinson's Study Group. Improvement of motor function in early Parkinson disease by safinamide. Neurology. 2004;63:746-748.
36. Stocchi F, Borgohain R, Onofrj M, et al. A randomized, double-blind, placebo-controlled trial of safinamide as add-on therapy in early Parkinson's disease patients. Mov Disord. 2012;27(1):106-112.

37. Schapira AH, Stocchi F, Borgohain R, et al; Study 017 Investigators. Long-term efficacy and safety of safinamide as add-on therapy in early Parkinson's disease. Eur J Neurol. 2013;20:271-280.

38. Barone P, Fernandez HH, Ferreira J, et al. Safinamide as an add-on therapy to a stable dose of a single dopamine agonist: results from a randomized, placebo-controlled, 24-week multicenter trial in early idiopathic Parkinson disease (PD) patients (MOTION Study) [abstract]. Neurology. 2013;80(Meeting Abstracts 1).

39. Onofrj M, Szasz J, Stanzione P. Randomized trial of safinamide add-on to levodopa in Parkinson's disease with motor fluctuations. Mov Disord. 2014;29(2):229-237

40. Borgohain R, Szasz J, Stanzione P et al. Randomized trial of safinamide add-on to levodopa in Parkinson's disease with motor fluctuations. Mov Disord. 2014;29(2):229-237.

41. Schapira AH, Fox SH, Hauser RA, et al. Safinamide add on to L-Dopa: a randomized, placebo-controlled, 24-week global trial in patients with Parkinson's disease (PD) and motor fluctuations (SETTLE) [abstract] Neurology. 2013;80(Meeting Abstracts 1)
Journal of Parkinsonism \& Restless Legs Syndrome

\section{Publish your work in this journal}

Journal of Parkinsonism and Restless Legs Syndrome is an online, open access, peer-reviewed journal. The journal publishes review articles, historical reviews, original research articles, case reports, letters to the editor, clinical teaching cases, neuroradiology highlights, neuropathology highlights, neuropsychiatry highlights, autobiographies, conference

\section{Dovepress}

proceedings, abstracts and book reviews. The manuscript management system is completely online and includes a very quick and fair peerreview system, which is all easy to use. Visit http://www.dovepress.com/ testimonials.php to read real quotes from published authors. 